\title{
Controlling UAV networks in uncertain environments
}

Unmanned Aerial Vehicles (UAVs) look set to play an increasingly important part in years, but the technology faces a unique set of challenges before it can become fully integrated into our infrastructures. In her University of Texas in Arlington considers how these challenges can be addressed; paying particular attention to how networks of UAVs can carry out tasks together. The insights gathered by her team already promise to transform the ways

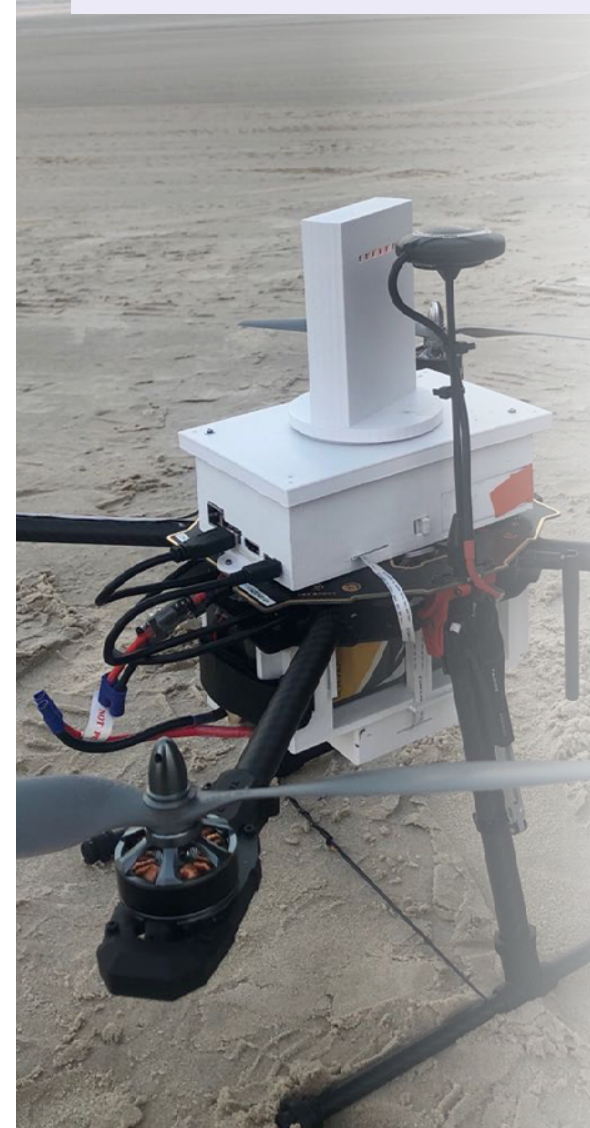

$n$ recent years, UAVs have gone from being little more than toys for taking unique holiday photos, to a technology which is rapidly becoming an important part of our society. Now, they look set to become key elements of an emerging group of technologies named cyberphyical systems, "hose bed aviours the physical world and computer algorithms Leading reseach into UAVs is Dr Yan Wan, Associate Professor of Electrical Engineering at the University of Texas at Arlington. As Dr Wan explains, many researchers predict that with these advances UAVs could soon be crucial components of the 'internet of things' (IOT)-which links many of the systems we use in our everyday lives into an extensive, intelligent network of devices.

Dr Wan summarises, "Examples of civilian applications include unmanned aerial taxis, emergency response, traffic surveillance, precision agriculture, cargo transport, personal outdoor and infrastrcture helth me, land survey, integration is far from simple. In her research, Dr Wan aims to confront. the challenges which arise with the burgeoning technology; particularly in regard to how systems of many UAV can be used to complete the tasks to them.

\section{THE CHALLENGE OF MULTIPLE-} UAV NETWORKS

So far, UAV s have largely been used to carry out tasks by themselves. However recent research has shown that with the right controls in place, the vehicles have strength in numbers when it com to carrying out more complex tasks. achieve highly complicated missions more effectively," says DrWan. "This requires aibon," says bringn, "This utilises UAV-UAV communication to share information, maintain safe manoeuvring, and coordinate to achieve time-

Despite such numerous advantages, network of devices which constantly talk o each other to exchange information, each other in continually changing environments, would be extremely difficut to realise. "Airborne networking unlike the networking of fixed sensors, mobile devices, and slow-moving vehicles, is very challenging because of the high mobility, stringent safety requirements, and uncertain airspace environment," DrWan continues. To combat these issues, DrWan and her team approach the problem from anew angle-considering how certain aspects of UAV networks-previously seen as hinderances, could be used their advantage.

Previously, researchers have viewed in a network, and their mons. the network as being at odds with each other. The more the vehicles move around relative to each other. they claim the harder it must be for them to stay in touch. In contrast, $\mathrm{Dr}$ Wan has brought together a set of computational techniques which allow UAV networks to exploit these constraints and uncertainties; using them to gather information about their present situations. This project uses an innovative codesign approach that exploits the mutua benefits of networking and decentralised mobility control in an uncertain

environment," she says. Three distinct results highlight the successes of this approach - ach addressing a different
CHANGING DIRECTION IN UNCERTAIN ENVIRONMENTS

The first of these developments is called a random mobility modelling framework,' which takes account how individual UAVs must adopt highly

variable paths

as they carry

out their

missions.

While previous

studies have

Multiple UAVs can coordinate and achieve highly complicated missions more effectively.

ndom mobility models for ground vehicles and mobile users, these models do not capture UAV mobility physics, the as three-dimensional movement and the smoothness of trajectories.

Dr Wan and colleagues used an updated approach to consider how airborne objects are influenced over time as they move along threedimensional paths. In particular, they have considered how these trajectories must be continually adapted due to the random behaviours of ther surroundings. Such behaviour is directly comparable to real-life scenarios, wher UAVs are constantly buffeted by their varying missions and air movents to such as vertical thernals, and field of UAV design. "The smooth turn framework that we developed has been widely adopted for UAV network design and evaluation studies," Dr Wan explains. By simulating the behaviours of random create UAV networks which directly adapt their dynamics to work with of fighting against them.
ond OF DIMENSIONALITY Secondly, Dr Wan and her colleagues dealt with a long-standing puzzle facing many different types of uncertain complex networks: the curse of dimensionality. The problem "describes the exponential growth of

\section{BREAKING THE CURSE}

involves the evaluation of highimensional uncertainties. To solve the problem, the researchers developed Probabilistic Collocation Method' (M-PCM)-which introduces new computational techniques to randomly sample the properties of high-dimensional systems. When merged with a further 'orthoue, named M-PCN factorial design,' the uncertainties surrounding the behaviours of AV networks in $3 \mathrm{D}$ could finally be broken down.

"This solution breaks the curse to the accuracy and scalability of the optimal control of random systems, reinforcement-learning based control, and dynamic graphical games," DrW describes. Ultimately, the technique would allow for more effective ways to design and control systems of multiple UAVs, and to assess their performance in real time. Again, this development demonstrates how, rather than being a can be exploted to fall, randons decision in complex networks. 


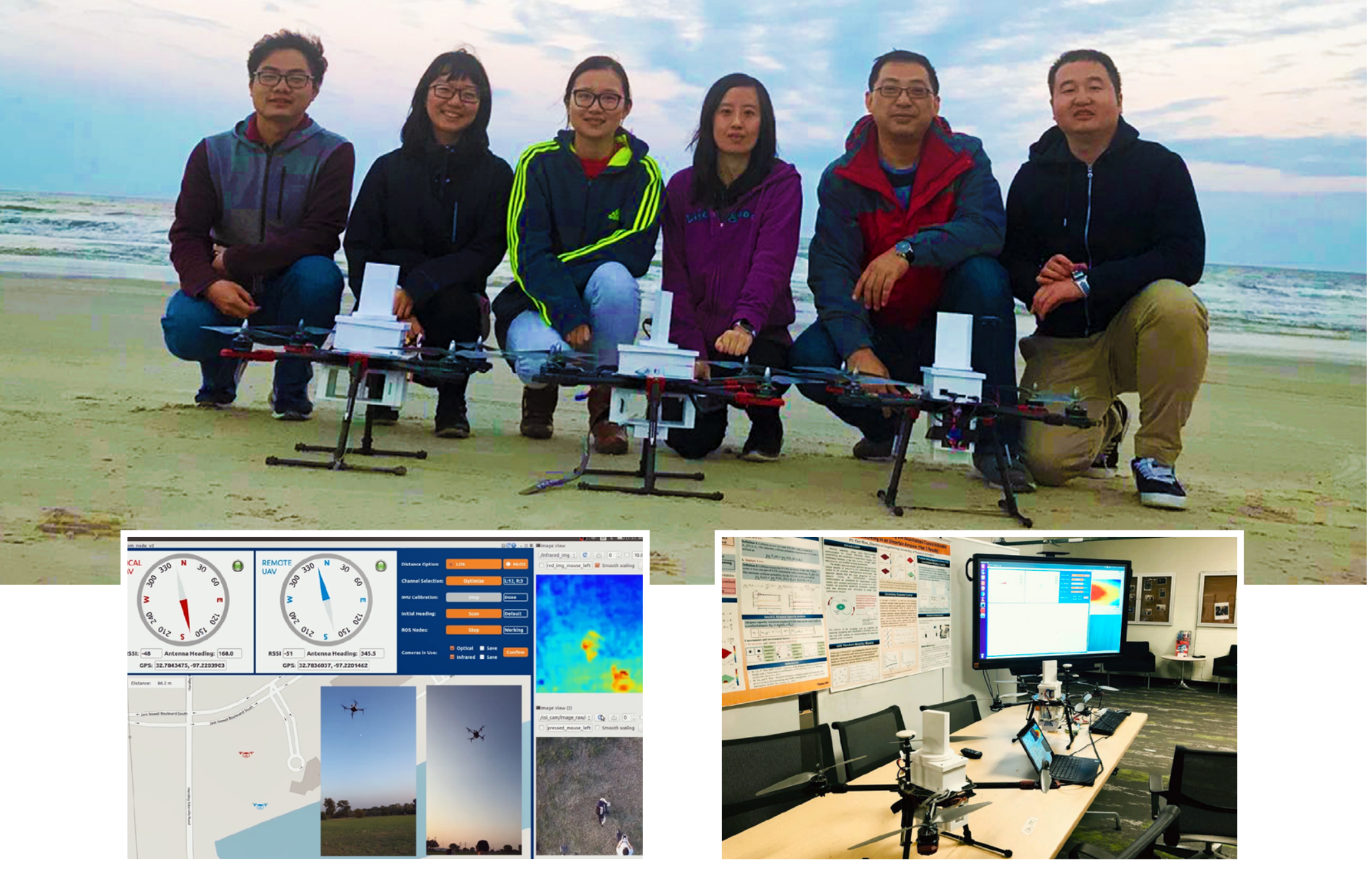

\section{LAYERED FORMATIONS} Finally, DrWan's team investigated how the effectiveness of communication and data transmission between UAV within a network can vary when the vehicles fly in different formations. Contrary to previous studies, they discovered that UAV networks don't perform at their best when arranged in egalitarian structures, have free rign over where they travel hive free reign over where they trave be more easily controlled and managed when the vehicles are arranged in neat layers, stacked on top of each other.

"We examined various network structures and found that the layered structures, which reside between centralised and decentralised structures, are very DrWan explains. "If designed properly, such layered structures can significanty reduce throughput while still allowing efficient completion of distributed con tasks." This insight was the final piece of the puzle when it came to the effective operation of UAV networks. Together

...rather than being a barrier to effective control, randomness can be exploited

to facilitate robust decision in complex networks.

most recent studies, the insights have the technology for the first time.

TRANSFORMING THE CAPABILTIES OF UAV NETWORKS

Where the inevitable uncertain characteristic of random systems have previously hindered communication and mobility in UAV networks, the results of DrWan and her colleagues clearly demonstrate how randomness can instead be embraced. When the right precautions are taken, even highly complex aerial networks of UAVs, all talking to each other in real time, would be able to seamlessly interact with the environments. DrWan pursued the transfer of the developed technolog to emergency response, where the communication service to assist first responders. Dr Wan concludes that her significant new advances in technology as a whole.

"The technological advances developed in this research will contribute to multiple fields, including mobile networking, edge computing, decentralised control, experiment design and general real-time decision making under uncertainty for cyber-physical systems, she says. Thanks to DrWa and her colleagues, the emerging looks set to enjoy a bright future-with in many of the systems operation which could an ease of technology of UAV networks now the potential to become an integral part

\section{Dr Yan Wan}

E: yan.wan@uta.edu T: +1 817-272-6838

\section{Research Objectives}

Dr Yan Wan's research interests lie in the modelling evaluation, and control of large-scale dynamical networks, control leacing control networking, uncertainty analysed algebraic graph theory, and their applications to unmanne, aerial vehicle (UAV) networking UAV traffic management, epidemic spread, complex information networks, and air traffic management.

\section{Detail}

416 Yates Street,

543 Nedderman Hall,

Electrical Engineering,

Arlington, TX

Bio

Dr Yan Wan is Associate Professor in the Electrical Engineering Department at the University of Texas at Arlington. She received her Ph.D. in Electrical Engineering from Washington State University in 2009 and postdoctora training at the University of California, Santa Barbara. Her research has led to over 160 publications and successful technology transfer outcomes. She has been recognised by several prestiglous awards, including the US I In

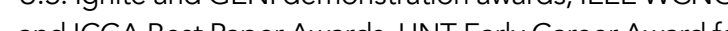
Research and Creativity, UTA STARS Award and Tech Titer of the Future - University Level Award.

Funding

National Science Foundation CAREER Program 1714519 and 1453722.

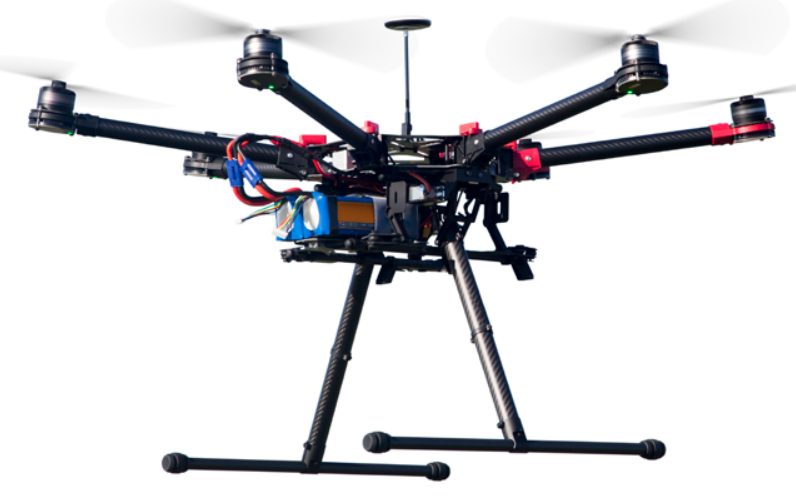

References

Xie, J., Wan, Y., Wang, B., Fu, S., Lu, K., Kim, J.H. (2018). A comprehensive 3-dimensional random mobility modeling framework for airborne networks. IEEE Access, 6,

Xie, J., Wan, Y., Mills, K., Filliben, J.J., Lewis, F.L. (2017). A scalable sampling method to high-dimensional uncertainties for optimal and reinforcement learning-based controls. DOl: 10.1109/LSYS $2017,2708598$.

Chen, J., Xie, J., Gu, Y., Li, S., Fu, S., Wan, Y., Lu, K. (2017). Long-range and broadband aerial communication using IEEE Transactions on Vehicular Technology, 66(12), 1079310805. DOI: https://doi.org/10.1109/TVT.2017.2723802.

Xie, J., Wan, Y., Mills, K., Filliben, J.J, Lei, Y., Lin, Z. (2019). M-PCM-OFFD: An effective output statistics estimation method for systems of high dimensional uncertainties subject to low-order parameter interactions. Mathematics and Computers in Simulation, 159, 93-118. DOl: 10.1016/j.matcom.2018.10.010.

Wan, Y., Yan, J., Lin, Z., Sheth, V., Das, S.K. (2019). On the structural perspective of computational effectiveness for quantized consensus in layered UAV networks. IEEE 101109 TCNS 20182813926

\section{Personal Response}

What is next for your research?

II We plan to extend our study to explore UAV traffic management, which is concerned with maintaining airspace safety and efficiency for dense operations of UAVs. While that we developed are important components of this problem, UAV traffic management covers much boarder, scales, such as airspace capacity management, UAV path scheduling, infrastructure support, human system contingency management, security, verification, and various operational challenges. Ultimately, we work toward a safe complements our existing ground

traffic systems. 